\title{
Minimal-Learning-Parameter Technique Based Adaptive Neural Sliding Mode Control of MEMS Gyroscope
}

\author{
Bin Xu and Pengchao Zhang \\ Shaanxi Provincial Key Laboratory of Industrial Automation, Shaanxi University of Technology, Hanzhong, \\ Shaanxi 723000, China \\ Correspondence should be addressed to Bin Xu; smileface.binxu@gmail.com
}

Received 15 May 2017; Accepted 27 June 2017; Published 26 July 2017

Academic Editor: Yanan Li

Copyright (C) 2017 Bin Xu and Pengchao Zhang. This is an open access article distributed under the Creative Commons Attribution License, which permits unrestricted use, distribution, and reproduction in any medium, provided the original work is properly cited.

\begin{abstract}
This paper investigates an adaptive neural sliding mode controller for MEMS gyroscopes with minimal-learning-parameter technique. Considering the system uncertainty in dynamics, neural network is employed for approximation. Minimal-learningparameter technique is constructed to decrease the number of update parameters, and in this way the computation burden is greatly reduced. Sliding mode control is designed to cancel the effect of time-varying disturbance. The closed-loop stability analysis is established via Lyapunov approach. Simulation results are presented to demonstrate the effectiveness of the method.
\end{abstract}

\section{Introduction}

Recently, MEMS gyroscopes have been drawing growing attention because they intend to employ advanced control approaches to realize trajectories tracking and to handle system parametric uncertainties and disturbances. These intelligent control methods improve the performance of gyroscopes, so that the applications of MEMS gyroscopes are expanded. With the vigorous development of nonlinear system control methods [1-8], a variety of gyroscopic modal control methods emerged.

In $[9,10]$, one adaptive operation strategy is presented to control the MEMS $z$-axis gyroscope, which offers a larger operational bandwidth, absence of zero-rate output, self-calibration, and large robustness to parameter variations caused by fabrication defects and ambient conditions. In [11, 12], the sliding mode control is proposed to handle the vibrating proof mass, which achieves better estimation of the unknown angular velocity than conventional model reference adaptive feedback controller. Since then, in the presence of significant uncertainties, the regulated model-based and non-modelbased sliding model control approaches are presented to improve tracking control of the drive and sense modes of the vibratory gyroscope in [13]. In [14], an adaptive tracking controller with a proportional and integral sliding surface is proposed.

Neural network has an inherent ability to learn and approximate nonlinear functions $[15,16]$, which can be utilized for unstructured uncertainties. Thus, an adaptive control strategy using radial basis function (RBF) network/Fuzzy Logic System compensator is presented for robust tracking of MEMS gyroscope in the presence of model uncertainties and external disturbances to compensate such system nonlinearities and improve the tracking performance in [17-19]. However, in practical application, large amount of update parameters results in the computation burden of online learning. In [20], the minimal-learning-parameter technique is further incorporated into the high gain observer to greatly reduce the online computation burden.

Inspired by the above-mentioned discussions on designing intelligent controllers and reducing the number of online parameters, this paper will focus on constructing the new control scheme for MEMS gyroscopes to suppress the system parametric uncertainties and disturbances. The main contribution of this paper is that a single parameter is employed to replace weight matrix, which significantly reduces the computation burden. 


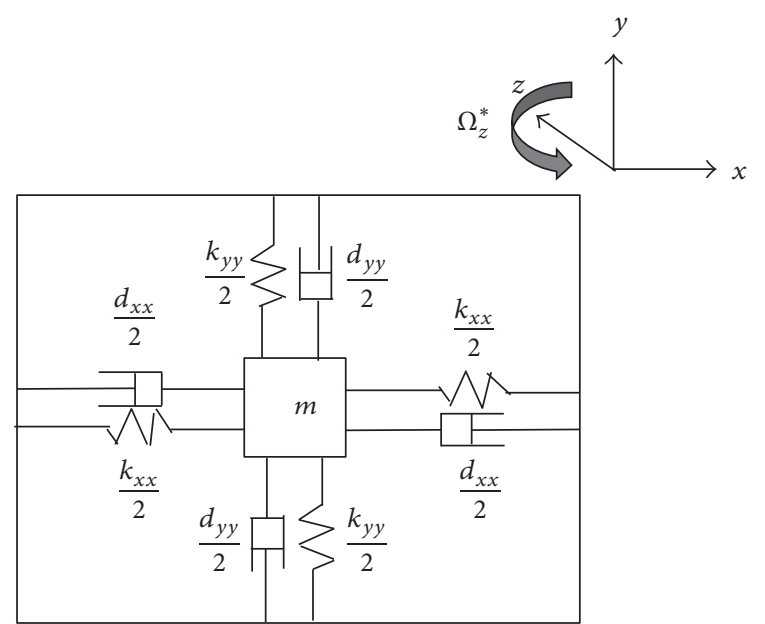

FIGURE 1: The basic principle diagram of $z$-axis MEMS gyroscope.

The structure of this paper is organized as follows. Section 2 formulates the dynamics of MEMS gyroscope. Section 3 studies the neural network of lumped parametric uncertainties. In Section 4, an adaptive neural sliding mode control strategy using the minimal-learning-parameter technique is designed and stability analysis is discussed. Numerical simulations are investigated to verify the superiority of the proposed approach in Section 5. Conclusions are given in Section 6.

\section{Problem Formulation}

2.1. Dynamics of MEMS Gyroscope. As Figure 1 shows, the ideal MEMS gyroscope is a quality-stiffness-damping system. Considering the mechanical coupling caused by manufacturing defects, the dynamics of MEMS gyroscopes can be expressed as

$$
\begin{aligned}
& m \ddot{x}+d_{x x} \dot{x}+\left(d_{x y}-2 m \Omega_{z}^{*}\right) \dot{y}+\left(k_{x x}-m \Omega_{z}^{*^{2}}\right) x \\
& +k_{x y} y=u_{x}^{*}, \\
& m \ddot{y}+d_{x x} \dot{y}+\left(d_{x y}+2 m \Omega_{z}^{*}\right) \dot{x}+\left(k_{y y}-m \Omega_{z}^{*^{2}}\right) y \\
& +k_{x y} x=u_{y}^{*},
\end{aligned}
$$

where $m$ represents the mass of proof mass, $\Omega_{z}^{*}$ represents the input angular velocity, $x$ and $y$ represent the system generalized coordinates, $d_{x x}$ and $d_{y y}$ represent damping terms, $d_{x y}$ represents asymmetric damping term, $k_{x x}$ and $k_{y y}$ represent spring terms, $k_{x y}$ represents asymmetric spring terms, and $u_{x}^{*}$ and $u_{y}^{*}$ represent the control forces.

In (1), the damping terms are affected by atmospheric pressure and spring terms are affected by ambient temperature. That means

$$
\begin{aligned}
& d_{x x}=d_{x 0}+\Delta d_{x x}, \\
& d_{y y}=d_{y 0}+\Delta d_{y y},
\end{aligned}
$$

$$
\begin{aligned}
& k_{x x}=k_{x 0}+\Delta k_{x x}, \\
& k_{y y}=k_{y 0}+\Delta k_{y y}, \\
& k_{x y}=k_{x y 0}+\Delta k_{x y},
\end{aligned}
$$

where $d_{x 0}, d_{y 0}$, and $d_{x y 0}$ are the damping terms of MEMS gyroscope in normal atmospheric pressure, $k_{x 0}, k_{y 0}$, and $k_{x y 0}$ are the spring terms of MEMS gyroscope in room temperature environment, $\Delta d_{x x}, \Delta d_{y y}$, and $\Delta d_{x y}$ are the deviation in damping coefficients due to the changes of atmospheric pressure, and $\Delta k_{x x}, \Delta k_{y y}$, and $\Delta k_{x y}$ are the deviation of damping coefficients because of the changes of ambient temperature.

Dividing both sides of (1) by reference mass $m$, reference frequency $\omega_{o}^{2}$, and reference length $q_{o}$, the dynamics can be derived as

$$
\begin{aligned}
\frac{\mathbf{q}^{*}}{q_{o}} & +\frac{\mathbf{D}^{*}}{m \omega_{o}} \frac{\mathbf{q}^{*}}{q_{o}}+2 \frac{\mathbf{s}^{*}}{\omega_{o}} \frac{\mathbf{q}^{*}}{q_{o}}-\frac{\Omega_{z}^{*^{2}}}{\omega_{o}^{2}} \frac{\mathbf{q}^{*}}{q_{o}}+\frac{\mathbf{K}^{*}}{m \omega_{o}^{2}} \frac{\mathbf{q}^{*}}{q_{o}} \\
& =\frac{\mathbf{u}^{*}}{m \omega_{o}^{2} q_{o}},
\end{aligned}
$$

where $\mathbf{q}^{*}=\left[\begin{array}{l}x \\ y\end{array}\right], \mathbf{u}^{*}=\left[\begin{array}{l}u_{x}^{*} \\ u_{y}^{*}\end{array}\right], \mathbf{D}^{*}=\left[\begin{array}{ll}d_{x x} & d_{x y} \\ d_{x y} & d_{y y}\end{array}\right], \mathbf{S}^{*}=\left[\begin{array}{cc}0 & -\Omega_{z}^{*} \\ \Omega_{z}^{*} & 0\end{array}\right]$, and $\mathbf{K}^{*}=\left[\begin{array}{ll}k_{x x} & k_{x y} \\ k_{x y} & k_{y y}\end{array}\right]$.

Define new parameters as $\mathbf{q}=\mathbf{q}^{*} / q_{o}, \mathbf{u}=\mathbf{u}^{*} / m \omega_{o}^{2} q_{o}$, $\Omega_{z}=\Omega_{z}^{*} / \omega_{o}, \mathbf{D}=\mathbf{D}^{*} / m \omega_{o}, \mathbf{K}=\mathbf{K}^{*} / m \omega_{o}^{2}$, and $\mathbf{S}=-\mathbf{S}^{*} / \omega_{o}$.

Then, (3) has the following form:

$$
\ddot{\mathbf{q}}=(2 \mathbf{S}-\mathbf{D}) \dot{\mathbf{q}}+\left(\Omega_{z}^{2}-\mathbf{K}\right) \mathbf{q}+\mathbf{u},
$$

where $\mathbf{D}=\mathbf{D}_{0}+\Delta \mathbf{D}, \mathbf{K}=\mathbf{K}_{0}+\Delta \mathbf{K}$ and $\mathbf{D}_{0}=$ $\left[\begin{array}{ll}d_{x 0} / m \omega_{o} & d_{x y} / m \omega_{o} \\ d_{x y 0} / m \omega_{o} & d_{y 0} / m \omega_{o}\end{array}\right], \quad \mathbf{K}_{0}=\left[\begin{array}{cc}k_{x 0} / m \omega_{o}^{2} & k_{x y 0} / m \omega_{o}^{2} \\ k_{x y 0} / m \omega_{o}^{2} & k_{y 0} / m \omega_{o}^{2}\end{array}\right], \Delta \mathbf{D}=$ $\left[\begin{array}{l}\Delta d_{x x} / m \omega_{o} \Delta d_{x y} / m \omega_{o} \\ \Delta d_{x y} / m \omega_{o} \Delta d_{y y} / m \omega_{o}\end{array}\right]$, and $\Delta \mathbf{K}=\left[\begin{array}{l}\Delta k_{x x} / m \omega_{o}^{2} \Delta k_{x y} / m \omega_{o}^{2} \\ \Delta k_{x y} / m \omega_{o}^{2} \Delta k_{y y} / m \omega_{o}^{2}\end{array}\right]$.

With the external disturbances caused by compound maneuvers, (4) is replaced by

$$
\begin{aligned}
\ddot{\mathbf{q}}= & \left(2 \mathbf{S}-\mathbf{D}_{0}-\Delta \mathbf{D}\right) \dot{\mathbf{q}}+\left(\Omega_{\mathbf{z}}^{2}-\mathbf{K}_{0}-\Delta \mathbf{K}\right) \mathbf{q}+\mathbf{C u} \\
& +\mathbf{d}(\mathbf{t}),
\end{aligned}
$$

where $\mathbf{d}(\mathbf{t})$ is the external disturbance and $\mathbf{d}_{\mathbf{m}}=\sup |\mathbf{d}(\mathbf{t})|$.

Define lumped parametric uncertainties as

$$
\mathbf{P}(\dot{\mathbf{q}}, \mathbf{q})=-\Delta \mathbf{D} \dot{\mathbf{q}}-\Delta \mathbf{K q}
$$

Equation (5) can be written as

$$
\ddot{\mathbf{q}}=\mathbf{A} \dot{\mathbf{q}}+\mathbf{B q}+\mathbf{C u}+\mathbf{P}(\dot{\mathbf{q}}, \mathbf{q})+\mathbf{d}(\mathbf{t}),
$$

where $\mathbf{A}=2 \mathbf{S}-\mathbf{D}_{0}$ and $\mathbf{B}=\Omega_{\mathbf{z}}^{2}-\mathbf{K}_{0}$. 
Remark 1. In order to make the uncertainty of MEMS gyroscope depicted in (6) controllable, there must be unknown matrices of appropriate dimensions $\mathbf{G}, \mathbf{H}$, and $\mathbf{p}(\mathbf{t})$ such that $\Delta \mathbf{A}=\mathbf{C G}, \Delta \mathbf{B}=\mathbf{C H}$, and $\mathbf{d}(\mathbf{t})=\mathbf{C p}(\mathbf{t})$. And $\mathbf{C}$ is selected as $\mathrm{C}=\left[\begin{array}{ll}1 & 0 \\ 0 & 1\end{array}\right]$.

2.2. Control Goal. The control goal of this paper is to design a controller to steer the position $q$ and the speed $\dot{q}$ to the desired trajectories $\mathbf{q}_{\mathbf{m}}(t)=\left[\begin{array}{ll}x_{m} & y_{m}\end{array}\right]^{T}$ and $\dot{\mathbf{q}}_{\mathbf{m}}(t)=\left[\begin{array}{ll}\dot{x}_{m} & \dot{y}_{m}\end{array}\right]^{T}$. Besides, the minimal-learning-parameter technique is further incorporated into the estimation of lumped parametric uncertainties $\mathbf{P}(\dot{\mathbf{q}}, \mathbf{q})$.

\section{Brief Description of RBF Neural Network}

A neural network is established to approximate the lumped parametric uncertainties $\mathbf{P}(\dot{\mathbf{q}}, \mathbf{q})$, which can be expressed as

$$
\widehat{\mathbf{P}}(\dot{\mathbf{q}}, \mathbf{q} \mid \boldsymbol{\theta})=\widehat{\boldsymbol{\theta}}^{\mathrm{T}} \boldsymbol{\mu}(\dot{\mathbf{q}}, \mathbf{q}),
$$

where $\widehat{\boldsymbol{\theta}} \subseteq \mathbf{R}^{\mathbf{n}}$ is the adjustable parameter matrix, $\boldsymbol{\mu}(\dot{\mathbf{q}}, \mathbf{q})$ is a nonlinear vector function of the inputs, and the RBF has the form

$$
\boldsymbol{\mu}_{\mathbf{i}}=\exp \left(-\frac{\left\|\mathbf{q}-\mathbf{q}_{\mathbf{m i}}\right\|}{2 \sigma_{i}^{2}}\right), \quad i=1,2, \ldots, n,
$$

where $\mathbf{q}_{\mathbf{m i}}$ is an $n$-dimensional vector representing the center of the $i$ th basis function and $\sigma_{i}$ is the variance representing the spread of the basis function.

Suppose that $\boldsymbol{\theta}^{*}$ are the optimal weight parameters; parametric uncertainties could be reexpressed as

$$
\mathbf{P}(\dot{\mathbf{q}}, \mathbf{q})=\theta^{* T} \boldsymbol{\mu}(\dot{\mathbf{q}}, \mathbf{q})+\varepsilon
$$

where $\varepsilon$ is the optimal estimation error of RBF neural network and $\boldsymbol{\varepsilon}_{n}=\sup |\boldsymbol{\varepsilon}|$.

Thus, the estimation error can be written as

$$
\begin{aligned}
\mathbf{P}-\widehat{\mathbf{P}} & =\boldsymbol{\theta}^{* \mathrm{~T}} \boldsymbol{\mu}(\dot{\mathbf{q}}, \mathbf{q})+\boldsymbol{\varepsilon}-\widehat{\boldsymbol{\theta}}^{\mathrm{T}} \boldsymbol{\mu}(\dot{\mathbf{q}}, \mathbf{q}) \\
& =-\widetilde{\boldsymbol{\theta}}^{\mathrm{T}} \boldsymbol{\mu}(\dot{\mathbf{q}}, \mathbf{q})+\boldsymbol{\varepsilon}
\end{aligned}
$$

where $\widetilde{\boldsymbol{\theta}}=\widehat{\boldsymbol{\theta}}-\boldsymbol{\theta}^{*}$

\section{Adaptive Sliding Mode Control with Minimal-Learning-Parameter Technique}

Define the system tracking error as

$$
\mathbf{e}(\mathbf{t})=\mathbf{q}(\mathbf{t})-\mathbf{q}_{\mathbf{m}}(\mathbf{t}) .
$$

Select the sliding mode function as

$$
\mathbf{s}(\mathbf{t})=\dot{\mathbf{e}}(\mathbf{t})+\boldsymbol{\beta} \mathbf{e}(\mathbf{t}),
$$

where $\boldsymbol{\beta}$ is satisfied with Hurwitz condition.

The derivative of $\mathbf{s}(\mathbf{t})$ is

$$
\begin{aligned}
\dot{\mathbf{s}}(\mathbf{t})= & \ddot{\mathbf{e}}(\mathbf{t})+\beta \dot{\mathbf{e}}(\mathbf{t})=\left[\ddot{\mathbf{q}}(\mathbf{t})-\ddot{\mathbf{q}}_{\mathbf{m}}(\mathbf{t})\right]+\beta \dot{\mathbf{e}}(\mathbf{t}) \\
= & \mathbf{A} \dot{\mathbf{q}}+\mathbf{B q}+\mathbf{C u}+\mathbf{P}(\dot{\mathbf{q}}, \mathbf{q})+\mathbf{d}(\mathbf{t})-\ddot{\mathbf{q}}_{\mathbf{m}}(\mathbf{t}) \\
& +\beta \dot{\mathbf{e}}(\mathbf{t}) .
\end{aligned}
$$

Define $\phi=\left\|\boldsymbol{\theta}^{*}\right\|^{2}$ and the estimation error is $\widetilde{\phi}=\widehat{\phi}-\phi$, where $\widehat{\phi}$ is the estimation of $\phi$.

Assume that $\dot{\mathbf{s}}=\mathbf{0}$; according to (14), controller could be designed as

$$
\begin{aligned}
\mathbf{u} & =\mathbf{C}^{-1}\left[\ddot{\mathbf{q}}_{\mathbf{m}}-\mathbf{A} \dot{\mathbf{q}}-\mathbf{B q}-\frac{1}{2} \mathbf{s} \widehat{\phi} \boldsymbol{\mu}^{\mathrm{T}} \boldsymbol{\mu}-\widehat{\boldsymbol{\eta}}_{\mathbf{m}} * \operatorname{sgn}(\mathbf{s})\right. \\
& -\beta \dot{\mathbf{e}}-\mathbf{K s}],
\end{aligned}
$$

where $\boldsymbol{\eta}_{\mathrm{m}}=\sup |\boldsymbol{\eta}|, \boldsymbol{\eta}=\boldsymbol{\varepsilon}+\mathbf{d}(\mathbf{t}), \widehat{\boldsymbol{\eta}}_{\mathrm{m}} * \operatorname{sgn}(\mathbf{s})$ is Hadamard product item, and $-\mathbf{C}^{-1} \mathbf{K s}$ is a robust item.

Remark 2. Compared with traditional results of MEMS control [15-17], in this paper, minimal-learning-parameter technique is employed for controller design to reduce computation burden.

Remark 3. When strong time-varying disturbances exist, the boundary layer is bigger than before and the estimation errors are increased.

The adaptive law of single parameter $\widehat{\phi}$ can be designed as

$$
\dot{\hat{\phi}}=\frac{\gamma}{2} \mathbf{s}^{\mathbf{T}} \mathbf{s} \boldsymbol{\mu}^{T} \boldsymbol{\mu}-\kappa \gamma \widehat{\phi}
$$

where $\gamma>0$ and $\kappa>0$.

An adaptive item $\widehat{\boldsymbol{\eta}}_{m}$ is employed to estimate $\boldsymbol{\eta}_{m}$, and the estimated error is $\widetilde{\boldsymbol{\eta}}_{m}=\widehat{\boldsymbol{\eta}}_{m}-\boldsymbol{\eta}_{m}$. The adaptive law of $\widehat{\boldsymbol{\eta}}_{m}$ is selected as

$$
\dot{\widehat{\boldsymbol{\eta}}}_{m}=\tau\left(|\mathbf{s}|-\alpha \widehat{\boldsymbol{\eta}}_{m}\right),
$$

where $\tau>0$ and $\alpha>0$.

Substitute (15) into (14):

$$
\begin{aligned}
& \dot{\mathbf{s}}(\mathbf{t})=\left[\ddot{\mathbf{q}}_{\mathbf{m}}-\mathbf{A} \dot{\mathbf{q}}-\mathbf{B q}-\frac{1}{2} \mathbf{s} \widehat{\phi} \boldsymbol{\mu}^{\mathrm{T}} \boldsymbol{\mu}-\widehat{\boldsymbol{\eta}}_{\mathbf{m}} * \operatorname{sgn}(\mathbf{s})\right. \\
& -\boldsymbol{\beta} \dot{\mathbf{e}}-\mathbf{K s}]+\mathbf{A} \dot{\mathbf{q}}+\mathbf{B q}+\mathbf{P}(\dot{\mathbf{q}}, \mathbf{q})+\mathbf{d}(\mathbf{t})-\ddot{\mathbf{q}}_{\mathbf{m}}(\mathbf{t}) \\
& +\boldsymbol{\beta} \dot{\mathbf{e}}(\mathbf{t})=-\frac{1}{2} \mathbf{s} \widehat{\phi} \boldsymbol{\mu}^{\mathrm{T}} \boldsymbol{\mu}-\widehat{\boldsymbol{\eta}}_{\mathbf{m}} * \operatorname{sgn}(\mathbf{s})+\left[\boldsymbol{\theta}^{* \mathrm{~T}} \boldsymbol{\mu}(\dot{\mathbf{q}}, \mathbf{q})\right. \\
& +\boldsymbol{\varepsilon}]+\mathbf{d}(\mathbf{t})-\mathbf{K s} .
\end{aligned}
$$


Theorem 4. Considering that the nonlinear system (7) is with parametric uncertainties and disturbances, if controller (15) and updating laws (16) and (17) are designed, then the boundedness of all the closed-loop system signals included in (19) can be guaranteed.

Proof. Lyapunov function is selected as

$$
L=\frac{1}{2} \mathbf{s}^{\mathrm{T}} \mathbf{s}+\frac{1}{2 \gamma} \widetilde{\phi}^{2}+\frac{1}{2 \tau} \widetilde{\boldsymbol{\eta}}_{\mathrm{m}}^{\mathrm{T}} \widetilde{\boldsymbol{\eta}}_{\mathrm{m}} .
$$

The derivative of Lyapunov function is

$$
\begin{aligned}
& \dot{L}=\mathbf{s}^{\mathrm{T}} \dot{\mathbf{s}}+\frac{1}{\gamma} \tilde{\phi} \dot{\hat{\phi}}+\frac{1}{\tau} \widetilde{\boldsymbol{\eta}}_{\mathbf{m}}^{\mathrm{T}} \dot{\hat{\boldsymbol{\eta}}}_{\mathbf{m}}=\mathbf{s}^{\mathrm{T}}\left[-\frac{1}{2} \mathbf{s} \widehat{\phi} \boldsymbol{\mu}^{T} \boldsymbol{\mu}-\widehat{\boldsymbol{\eta}}_{\mathrm{m}}\right. \\
& \left.* \operatorname{sgn}(\mathbf{s})+\left[\boldsymbol{\theta}^{* \mathbf{T}} \boldsymbol{\mu}(\dot{\mathbf{q}}, \mathbf{q})+\boldsymbol{\varepsilon}\right]+\mathbf{d}(t)-\mathbf{K s}\right]+\frac{1}{\gamma} \widetilde{\phi} \dot{\hat{\phi}} \\
& +\frac{1}{\tau} \widetilde{\boldsymbol{\eta}}_{\mathrm{m}}^{\mathrm{T}} \dot{\hat{\boldsymbol{\eta}}}_{\mathrm{m}}=-\frac{1}{2} \mathbf{s}^{\mathrm{T}} \mathbf{s} \widehat{\phi} \boldsymbol{\mu}^{T} \boldsymbol{\mu}+\mathbf{s}^{\mathrm{T}} \boldsymbol{\theta}^{* \mathrm{~T}} \boldsymbol{\mu}(\dot{\mathbf{q}}, \mathbf{q})+\mathbf{s}^{\mathrm{T}}[\boldsymbol{\varepsilon} \\
& \left.+\mathbf{d}(t)-\widehat{\boldsymbol{\eta}}_{\mathbf{m}} * \operatorname{sgn}(\mathbf{s})\right]-\mathbf{s}^{\mathrm{T}} \mathbf{K} \mathbf{s}+\frac{1}{\gamma} \tilde{\phi} \dot{\bar{\phi}}+\frac{1}{\tau} \widetilde{\boldsymbol{\eta}}_{\mathbf{m}}^{\mathrm{T}} \dot{\hat{\boldsymbol{\eta}}}_{\mathrm{m}} \\
& \leq-\frac{1}{2} \mathbf{s}^{\mathbf{T}} \mathbf{s} \widehat{\phi} \boldsymbol{\mu}^{T} \boldsymbol{\mu}+\frac{1}{2} \mathbf{s}^{\mathrm{T}} \mathbf{s} \phi \boldsymbol{\mu}^{T} \boldsymbol{\mu}+\frac{1}{2}+\mathbf{s}^{\mathbf{T}}\left[\boldsymbol{\eta}-\widehat{\boldsymbol{\eta}}_{\mathbf{m}}\right. \\
& * \operatorname{sgn}(\mathbf{s})]-\mathbf{s}^{\mathrm{T}} \mathbf{K} \mathbf{s}+\frac{1}{\gamma} \widetilde{\phi} \dot{\hat{\phi}}+\frac{1}{\tau} \widetilde{\boldsymbol{\eta}}_{\mathrm{m}}^{\mathrm{T}} \dot{\hat{\boldsymbol{\eta}}}_{\mathrm{m}} \leq-\frac{1}{2} \\
& \cdot \mathbf{s}^{\mathrm{T}} \mathbf{s} \widetilde{\phi} \boldsymbol{\mu}^{T} \boldsymbol{\mu}+\frac{1}{2}+\mathbf{s}^{\mathrm{T}}\left[\boldsymbol{\eta}-\boldsymbol{\eta}_{\mathrm{m}} * \operatorname{sgn}(\mathbf{s})+\boldsymbol{\eta}_{\mathrm{m}}\right. \\
& \left.* \operatorname{sgn}(\mathbf{s})-\widehat{\boldsymbol{\eta}}_{\mathbf{m}} * \operatorname{sgn}(\mathbf{s})\right]-\mathbf{s}^{\mathrm{T}} \mathbf{K} \mathbf{s}+\frac{1}{\gamma} \widetilde{\phi} \dot{\hat{\phi}}+\frac{1}{\tau} \widetilde{\boldsymbol{\eta}}_{\mathbf{m}}^{\mathrm{T}} \dot{\hat{\boldsymbol{\eta}}}_{\mathrm{m}} \\
& \leq \tilde{\phi}\left(-\frac{1}{2} \mathbf{s}^{\mathrm{T}} \mathbf{s}^{T} \boldsymbol{\mu}+\frac{1}{\gamma} \dot{\hat{\phi}}\right)+\frac{1}{2}+\mathbf{s}^{\mathrm{T}}\left[\left(\boldsymbol{\eta}_{\mathrm{m}}-\widehat{\boldsymbol{\eta}}_{\mathrm{m}}\right)\right. \\
& * \operatorname{sgn}(\mathbf{s})]-\mathbf{s}^{\mathrm{T}} \mathbf{K} \mathbf{s}+\frac{1}{\tau} \widetilde{\boldsymbol{\eta}}_{\mathrm{m}}^{\mathrm{T}} \dot{\hat{\boldsymbol{\eta}}}_{\mathrm{m}} .
\end{aligned}
$$

Substituting (16) and (17) into (20), the following inequality is obtained:

$$
\begin{aligned}
\dot{L} \leq & -\kappa \widetilde{\phi} \widehat{\phi}+\frac{1}{2}-\mathbf{s}^{\mathrm{T}} \mathbf{K s}-\mathbf{s}^{\mathrm{T}}\left[\widetilde{\boldsymbol{\eta}}_{\mathrm{m}} * \operatorname{sgn}(\mathbf{s})\right]+\widetilde{\boldsymbol{\eta}}_{\mathrm{m}}^{\mathrm{T}}|\mathbf{s}| \\
& -\alpha \widetilde{\boldsymbol{\eta}}_{\mathbf{m}}^{\mathrm{T}} \widehat{\boldsymbol{\eta}}_{\mathrm{m}} \leq-\frac{\kappa}{2}\left(\widetilde{\phi}^{2}-\phi^{2}\right)+\frac{1}{2}-\mathbf{s}^{\mathrm{T}} \mathbf{K s}-\alpha \widetilde{\boldsymbol{\eta}}_{\mathrm{m}}^{\mathrm{T}} \widehat{\boldsymbol{\eta}}_{\mathrm{m}} \\
= & -\frac{\kappa}{2} \widetilde{\phi}^{2}-\mathbf{s}^{\mathrm{T}} \mathbf{K s}+\left(\frac{\kappa}{2} \phi^{2}+\frac{1}{2}\right)-\alpha \widetilde{\boldsymbol{\eta}}_{\mathrm{m}}^{\mathrm{T}} \widehat{\boldsymbol{\eta}}_{\mathrm{m}},
\end{aligned}
$$

where $\kappa=2 \lambda / \gamma$ and $\lambda=\min \left\{\lambda_{1} \lambda_{2}\right\}, \lambda_{1}, \lambda_{2}$ are the eigenvalues of matrix K. Furthermore, we have

$$
\begin{aligned}
\dot{L} & \leq-\frac{\kappa}{2} \widetilde{\phi}^{2}-\mathbf{s}^{\mathrm{T}} \mathbf{K} \mathbf{s}+\left(\frac{\kappa}{2} \phi^{2}+\frac{1}{2}\right) \\
& \leq-\frac{\lambda}{\gamma} \widetilde{\phi}^{2}-\mathbf{s}^{\mathrm{T}} \mathbf{K s}+\left(\frac{\kappa}{2} \phi^{2}+\frac{1}{2}\right)
\end{aligned}
$$

$$
\begin{aligned}
& =-2 \lambda\left(\frac{1}{2 \gamma} \widetilde{\phi}^{2}+\frac{1}{2} \mathbf{s}^{\mathbf{T}} \mathbf{s}\right)+\left(\frac{\kappa}{2} \phi^{2}+\frac{1}{2}\right) \\
& =-2 \lambda\left(L-\frac{1}{2 \tau} \widetilde{\boldsymbol{\eta}}_{\mathbf{m}}^{\mathbf{T}} \widetilde{\boldsymbol{\eta}}_{\mathbf{m}}\right)+\left(\frac{\kappa}{2} \phi^{2}+\frac{1}{2}\right)=-2 \lambda L+Q,
\end{aligned}
$$

where $Q=(\lambda / \tau) \widetilde{\boldsymbol{\eta}}_{\mathbf{m}}^{\mathrm{T}} \widetilde{\boldsymbol{\eta}}_{\mathrm{m}}+(\kappa / 2) \phi^{2}+1 / 2$.

The solution of $(22)$ is

$$
L \leq \frac{Q}{2 \lambda}+\left(L(0)-\frac{Q}{2 \lambda}\right) e^{-2 \lambda t} .
$$

Then all the signals included in the Lyapunov function are bounded. This concludes the proof.

Remark 5. In practical application, the high-frequency switching control signals of MEMS gyroscopes result in serious chattering. Therefore, the saturation function sat $(s)$ is used to replace the sign function sgn(s) in (15). The saturation function $\operatorname{sat}(x)$ has the form

$$
\operatorname{sat}(x)= \begin{cases}1 & x>a \\ \frac{x}{a} & |x| \leq a \\ -1 & x<-a,\end{cases}
$$

where $a$ is a positive constant.

\section{Numerical Simulation}

In this section, the aforementioned control scheme of MEMS gyroscope is simulated, the controller of which is designed as (15), and the adaptive laws are proposed as (16) and (17).

Parameters of the MEMS gyroscope are as follows:

$$
\begin{aligned}
m & =0.57 \times 10^{-8} \mathrm{~kg}, \\
d_{x x} & =0.429 \times 10^{-6} \mathrm{Ns} / \mathrm{m}, \\
d_{y y} & =0.0429 \times 10^{-6} \mathrm{Ns} / \mathrm{m}, \\
d_{x y} & =0.0429 \times 10^{-6} \mathrm{Ns} / \mathrm{m}, \\
k_{x x} & =80.98 \mathrm{~N} / \mathrm{m}, \\
k_{y y} & =71.62 \mathrm{~N} / \mathrm{m}, \\
k_{x y} & =5 \mathrm{~N} / \mathrm{m}, \\
\Omega_{z} & =5.0 \mathrm{rad} / \mathrm{s} .
\end{aligned}
$$

Since the position of proof mass ranges within the scope of submillimeter and the natural frequency is generally in the range of kilohertz, the reference length is assumed as $q_{o}=10 \times 10^{-6} \mathrm{~m}$ and reference frequency is assumed as $\omega_{o}=1 \mathrm{kHz}$.

Suppose that the reference trajectories are $x_{m}=$ $\sin (1.71 t), \dot{x}_{m}=1.71 \cos (1.71 t), y_{m}=1.2 \sin (1.11 t)$, and $\dot{y}_{m}=1.2 \times 1.11 \cos (1.11 t)$, respectively. 

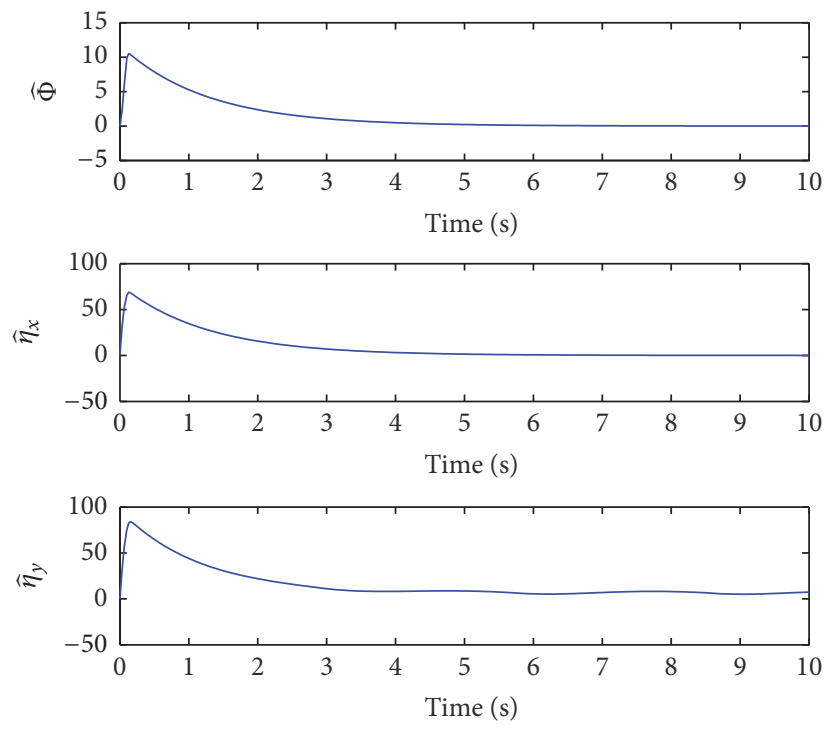

FIgURE 2: Adaptive signals.
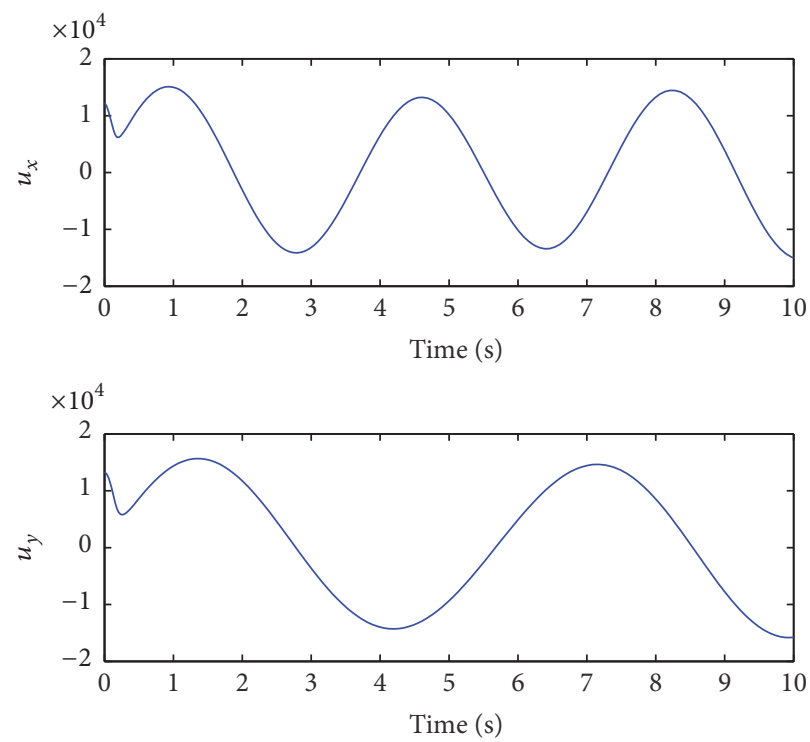

FIgURE 3: Control inputs.

Then set other simulation parameters as

$$
\begin{aligned}
\mathbf{A} & =\left[\begin{array}{cc}
-0.075 & 0.0025 \\
-0.0175 & -0.0075
\end{array}\right], \\
\mathbf{B} & =\left[\begin{array}{cc}
-14207 & -877 \\
-877 & -12564
\end{array}\right], \\
\mathbf{C} & =\left[\begin{array}{ll}
1 & 0 \\
0 & 1
\end{array}\right], \\
\mathbf{K} & =\left[\begin{array}{ll}
8 & 0 \\
0 & 4
\end{array}\right], \\
\boldsymbol{\beta} & =\left[\begin{array}{cc}
20 & 0 \\
0 & 15
\end{array}\right],
\end{aligned}
$$

$$
\begin{aligned}
\gamma & =80, \\
\kappa & =0.01, \\
\tau & =80, \\
\alpha & =0.01 .
\end{aligned}
$$

And select the initial state values of the system as $\left[\begin{array}{llll}0.8 & 0 & 1 & 0\end{array}\right]^{T}$. The centers of basis function for network are uniformly valued in $\left[\begin{array}{ll}-1 & 1\end{array}\right]$, and the spreads of the basis function are $\sigma_{i}=1$. The number of neural network nodes is chosen as 256 . The adaptive signals are presented in Figure 2, and the control inputs are shown in Figure 3. As 

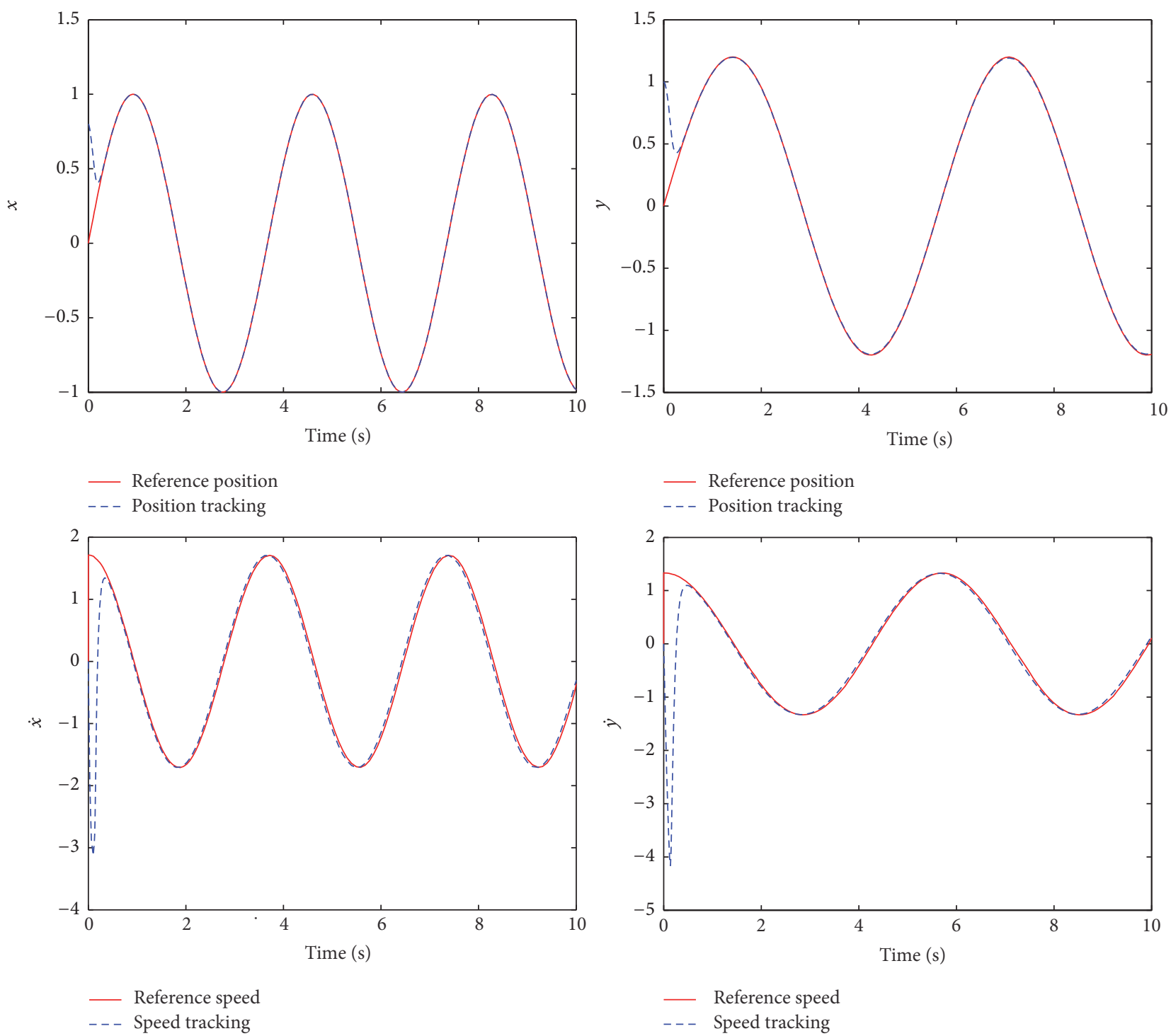

FIgUre 4: Position and speed trajectories.

depicted in Figure 4, the system using adaptive neural network sliding mode control with minimal-learning-parameter technique could track the reference signals very well. The position tracking errors and speed tracking errors are shown in Figure 5. Through the tracking simulations of MEMS gyroscope, the proposed approach has satisfying performance.

\section{Conclusions}

In this paper, an adaptive neural network sliding mode control strategy is proposed to compensate parametric uncertainties and external disturbances of MEMS gyroscopes. Based on Lyapunov criterion, system stability is guaranteed. With minimal-learning-parameter technique, the online computation burden is significantly reduced. Numerical simulations verify that the novel control scheme could track reference trajectories very well, which is similar to conventional adaptive neural network sliding mode control scheme. Therefore, the control scheme proposed in this paper could force the mass moves along reference trajectories, so that the performances of MEMS gyroscopes are improved.

For future work, more efficient learning methods [21-25] will be tested on the dynamics while the implementation for real systems will be analyzed.

\section{Conflicts of Interest}

The authors declare that they have no conflicts of interest. 

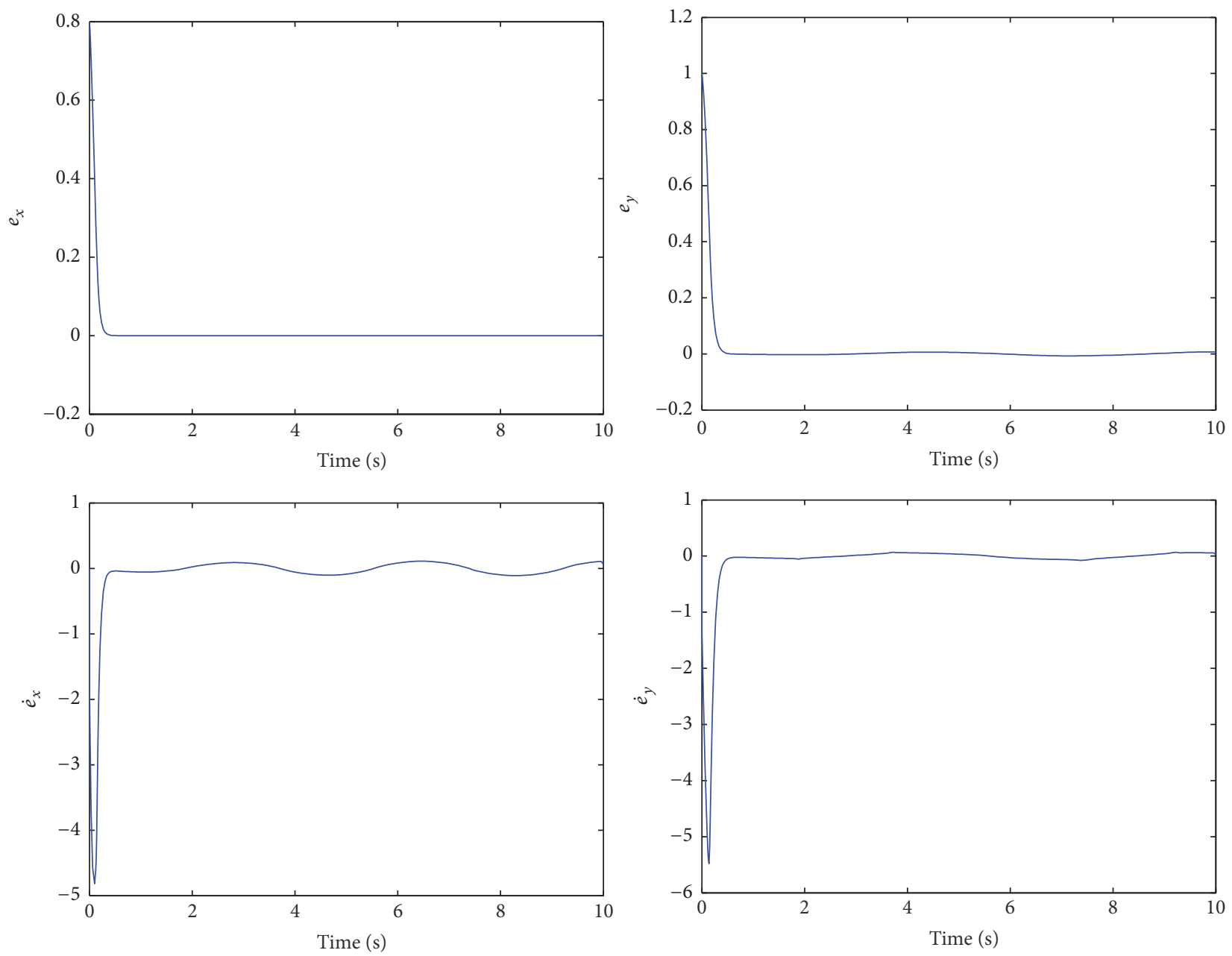

Figure 5: Position and speed tracking errors.

\section{Acknowledgments}

This work was supported by Industrial Science and Technology Key Project of Shaanxi Province (Grant no. 2016GY070) and Key Project of Shaanxi Province's Department of Education (Grant no. 2016JS017).

\section{References}

[1] M. Chen and S. S. Ge, "Adaptive neural output feedback control of uncertain nonlinear systems with unknown hysteresis using disturbance observer," IEEE Transactions on Industrial Electronics, vol. 62, no. 12, pp. 7706-7716, December 2015.

[2] B. Xu, D. Wang, Y. Zhang, and Z. Shi, "DOB based neural control of flexible hypersonic flight vehicle considering wind effects," IEEE Transactions on Industrial Electronics, vol. PP, no. 99, p. 1, 2017.

[3] B. Xu, "Composite learning finite-time control with application to quadrotors," IEEE Transactions on Systems, Man, and Cybernetics: Systems, vol. PP, no. 99, pp. 1-10, 2017.

[4] G.-X. Wen, C. L. Chen, Y.-J. Liu, and Z. Liu, "Neural-networkbased adaptive leader-following consensus control for secondorder non-linear multi-agent systems," IET Control Theory and Applications, vol. 9, no. 13, pp. 1927-1934, 2015.
[5] C. Yang, X. Wang, Z. Li, Y. Li, and C. Su, "Teleoperation control based on combination of wave variable and neural networks," IEEE Transactions on Systems, Man, and Cybernetics: Systems, vol. PP, no. 99, pp. 1-12, 2016.

[6] C. Yang, Y. Jiang, Z. Li, W. He, and C.-Y. Su, "Neural control of bimanual robots with guaranteed global stability and motion precision," IEEE Transactions on Industrial Informatics, vol. 13, no. 3, pp. 1162-1171, 2016.

[7] C. Yang, X. Wang, L. Cheng, and H. Ma, "Neural-learningbased telerobot control with guaranteed performance," IEEE Transactions on Cybernetics, vol. PP, no. 99, pp. 1-12, 2016.

[8] Q. Yang, S. Jagannathan, and Y. Sun, "Robust integral of neural network and error sign control of MIMO nonlinear systems," IEEE Transactions on Neural Networks and Learning Systems, vol. 26, no. 12, pp. 3278-3286, 2015.

[9] S. Park and R. Horowitz, "New adaptive mode of operation for MEMS gyroscopes," Journal of Dynamic Systems, Measurement and Control, Transactions of the ASME, vol. 126, no. 4, pp. 800810, 2004.

[10] R. P. Leland, "Adaptive control of a MEMS gyroscope using lyapunov methods," IEEE Transactions on Control Systems Technology, vol. 14, no. 2, pp. 278-283, 2006.

[11] J. D. John and T. Vinay, "Novel concept of a single-mass adaptively controlled triaxial angular rate sensor," IEEE Sensors Journal, vol. 6, no. 3, pp. 588-595, 2006. 
[12] C. Batur, T. Sreeramreddy, and Q. Khasawneh, "Sliding mode control of a simulated MEMS gyroscope," ISA Transactions, vol. 45, no. 1, pp. 99-108, 2006.

[13] A. Ebrahimi, "Regulated model-based and non-model-based sliding mode control of a MEMS vibratory gyroscope," Journal of Mechanical Science and Technology, vol. 28, no. 6, pp. 23432349, 2014.

[14] J. Fei and C. Batur, "A novel adaptive sliding mode control with application to MEMS gyroscope," ISA Transactions, vol. 48, no. 1, pp. 73-78, 2009.

[15] F.-J. Lin, S.-Y. Chen, and K.-K. Shyu, "Robust dynamic slidingmode control using adaptive RENN for magnetic levitation system," IEEE Transactions on Neural Networks, vol. 20, no. 6, pp. 938-951, 2009.

[16] L. Y. Wang, T. Y. Chai, and L. F. Zhai, "Neural-networkbased terminal sliding-mode control of robotic manipulators including actuator dynamics," IEEE Transactions on Industrial Electronics, vol. 56, no. 9, pp. 3296-3304, 2009.

[17] J. Fei and Y. Yang, "Adaptive neural compensation scheme for robust tracking of MEMS gyroscope," in Proceedings of the 2012 IEEE International Conference on Systems, Man, and Cybernetics, SMC 2012, pp. 1546-1551, October 2012.

[18] Y. P. Asad, A. Shamsi, and J. Tavoosi, "Backstepping-based recurrent type-2 fuzzy sliding mode control for MIMO systems (MEMS triaxial gyroscope case study)," International Journal of Uncertainty, Fuzziness and Knowledge-Based Systems, vol. 25, no. 2, pp. 213-233, 2017.

[19] J. X. Ren, R. Zhang, and B. Xu, "Adaptive fuzzy sliding mode control of MEMS gyroscope with finite time convergence," Journal of Sensors, vol. 2016, Article ID 1572303, 7 pages, 2016.

[20] B. Xu, Y. Fan, and S. Zhang, "Minimal-learning-parameter technique based adaptive neural control of hypersonic flight dynamics without back-stepping," Neurocomputing, vol. 164, no. 1-2, pp. 201-209, 2015.

[21] J. Yang, S. Li, and X. Yu, "Sliding-mode control for systems with mismatched uncertainties via a disturbance observer," IEEE Transactions on Industrial Electronics, vol. 60, no. 1, pp. 160-169, 2013.

[22] B. Xu and F. Sun, "Composite intelligent learning control of strict-feedback systems with disturbance," IEEE Transactions on Cybernetics, vol. PP, no. 99, pp. 1-12, 2017.

[23] M. Wang, Y. Zhang, and H. Ye, "Dynamic learning from adaptive neural control of uncertain robots with guaranteed full-state tracking precision," Complexity, In press.

[24] B. Luo, T. Huang, H.-N. Wu, and X. Yang, "Data-driven $\mathrm{H}_{\infty}$ control for nonlinear distributed parameter systems," IEEE Transactions on Neural Networks and Learning Systems, vol. 26, no. 11, pp. 2949-2961, 2015.

[25] B. Xu and P. Zhang, "Composite learning sliding mode control of flexible-link manipulator," Complexity, In press. 


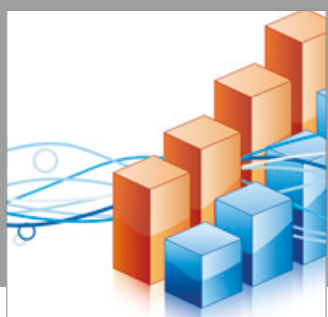

Advances in

Operations Research

vatersals

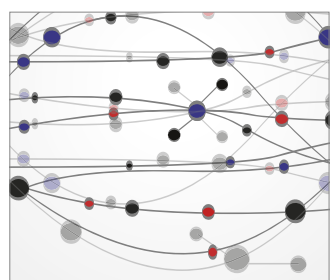

\section{The Scientific} World Journal
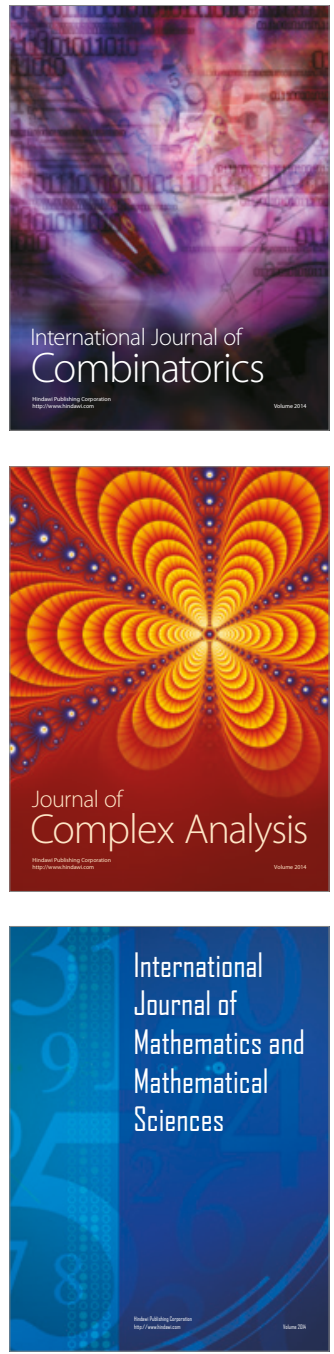
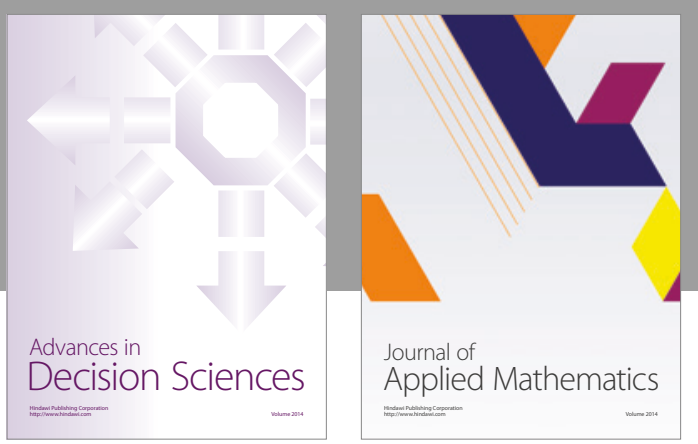

Algebra

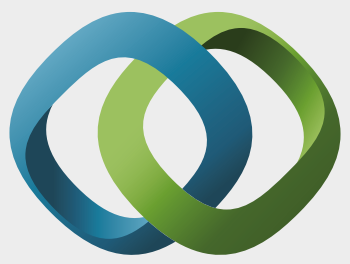

\section{Hindawi}

Submit your manuscripts at

https://www.hindawi.com
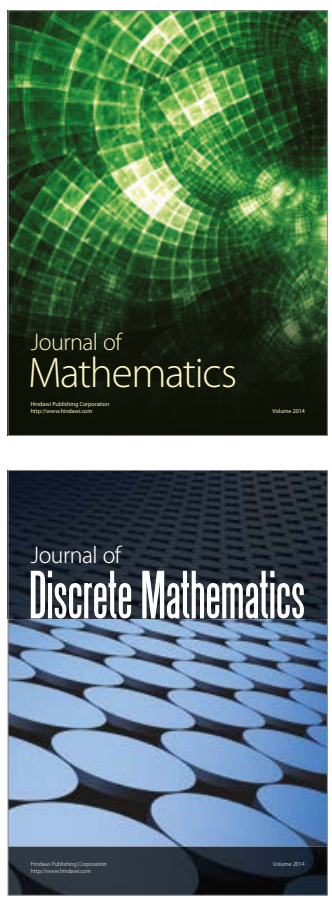

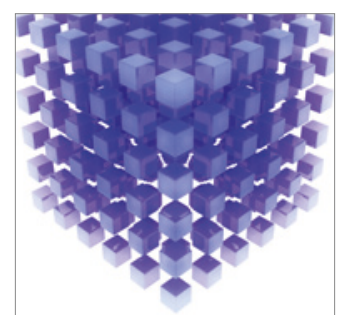

Mathematical Problems in Engineering
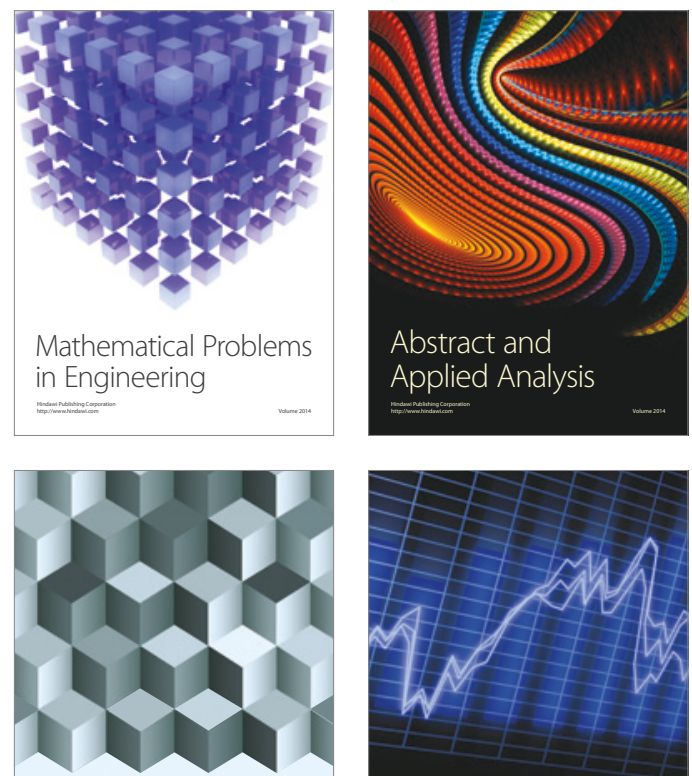

Journal of

Function Spaces

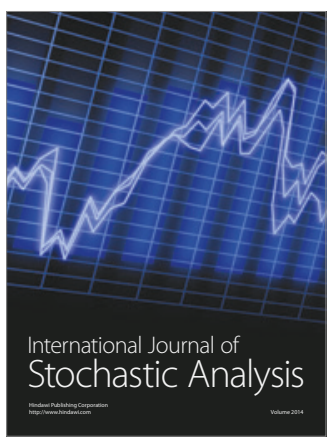

Probability and Statistics
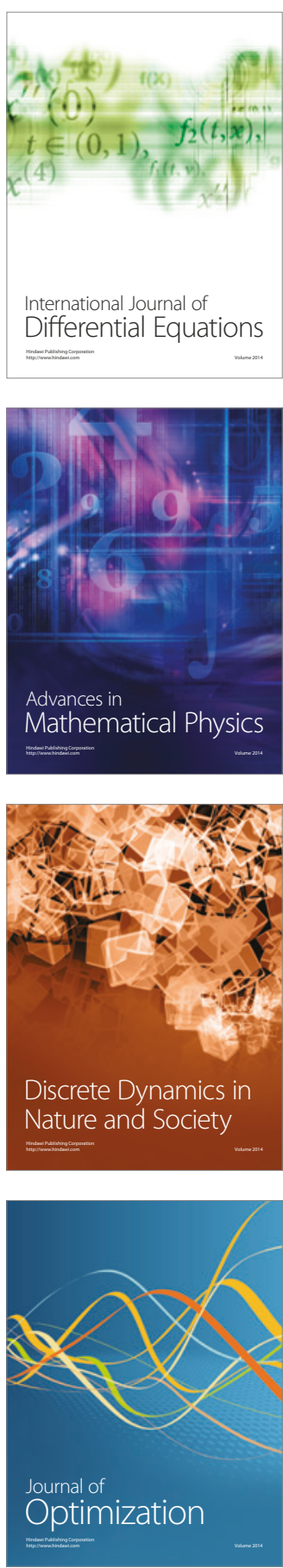